\title{
Affordances of visual representations and sense-making of science
}

\author{
Maurice M. W. Cheng
}

University of Waikato

Visual thinking is essential in the development of science. Visual representations are also indispensable when scientists disseminate their findings. This paper discusses the ways that research studies on visual representations can inform science learning and teaching. I start by discussing the ways that drawings, charts and graphics represent their referents, and hence highlighting the affordances of these visual representations. Then I discuss how these affordances make visual representations a valuable tool to support science teaching in formal and informal contexts and, in particular, how learning with media graphics can support the learning of Nature of Science for scientific literacy.

Keywords: teaching, learning, science capabilities, diagrammatic representations, data literacy

\section{Science and visual representations}

It is commonly accepted that observation is essential in science. Observation is important partly because it forms a basis for scientists to think through their disciplinary mind's eyes. For example, in the process of identifying the structure of atoms in the early 1900s, Marsden fired streams of positive-charged particles towards very thin gold foil - so thin that it was translucent (please see Figure 1a for an illustration) - and observed that while most of these particles passed through (which was expected, meaning much of an atom is empty space), a minute number of these particles bounced back (which was unexpected) (Figure 1b). Rutherford reasoned that this was possible only if there was a heavy positivecharged particle in each gold atom (Figure 2). Hence, he arrived at the idea that most of the mass of an atom was concentrated in a tiny nucleus (cf. with the then conjecture that the mass was evenly distributed in an atom). In developing his ideas of the structure of the gold atoms, Rutherford was likely to have mentally visualised what it could be like inside an atom that was consistent with the unexpected observation. In fact, much of science deals with objects that are too small (like atoms) or too vast (like our universe) and processes that are too fast (like explosions) or too slow (like the formation of waterfalls) to be observed directly by our naked eyes. It is through mental visual representations that these objects and processes are re-constructed based on available evidence or indirect observations. 


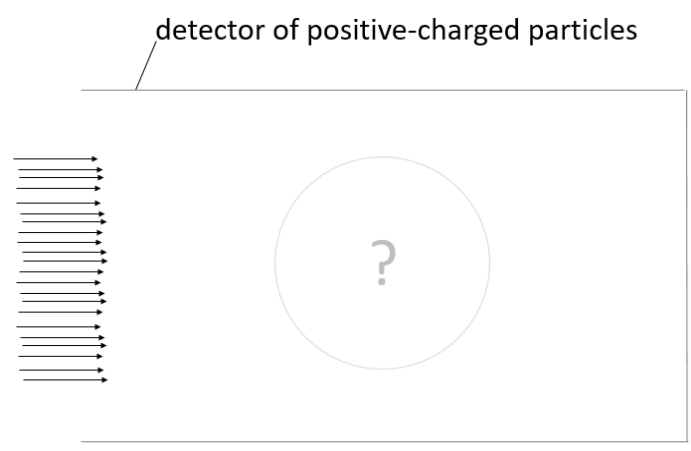

(a)

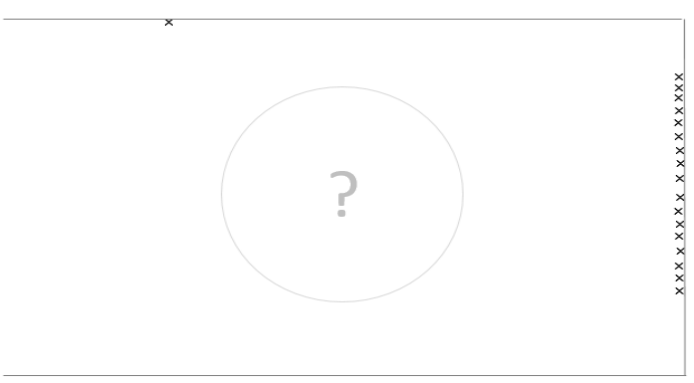

(b)

Figure 1. (a) The arrows represent the stream of positive-charged particles firing towards an atom of unknown internal structure. The chamber represents the detector of these particles. (b) Result: places where the detector found the positive-charged particles are marked with a cross. Note that a positive-charged particle bounced back

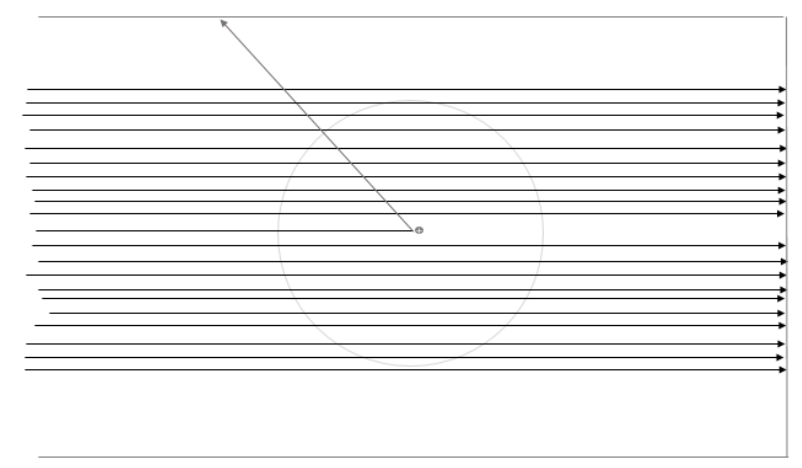

Figure 2. A visualisation of the paths of positive-charged particles. A positive-charged particle bounced back, suggesting that there must have been a small, heavy and positive-charged nucleus in the atom

Cognitive, social and historical studies of scientists suggest that scientists do not only rely on mental visual representations in making sense of physical phenomena, but also use drawings and charts, etc, as external visual representations in discussing ideas in laboratories, in developing ideas with their peers and disseminating their findings. For example, in recent times, science communicators in New Zealand created the graphic 'flattening the curve' to explain to the public how a lockdown could prevent the public healthcare system from being overstretched. In fact, the use of graphics to communicate and persuade can be dated back at least 160 years ago. While serving in the Crimean War, Florence Nightingale noticed that infectious diseases had claimed more lives than fatal injuries from battlefields, and that improved sanitary conditions significantly decreased hospital mortality. Although she was able to present the tabulated data to the British government, the numbers did not help to engage and convince her audience. Hence, she produced a 'rose diagram' that compared the casualties of infections (see the blue wedges in the right diagram of Figure 3) and injuries (red wedges in the same diagram), and 
changes in casualties of infections when there was an improvement in military hospital sanitation (the blue wedges in the left diagram being smaller than that in the right diagram, Figure 3). The diagram was shown to be a success in persuading reforms in hospital sanitation.

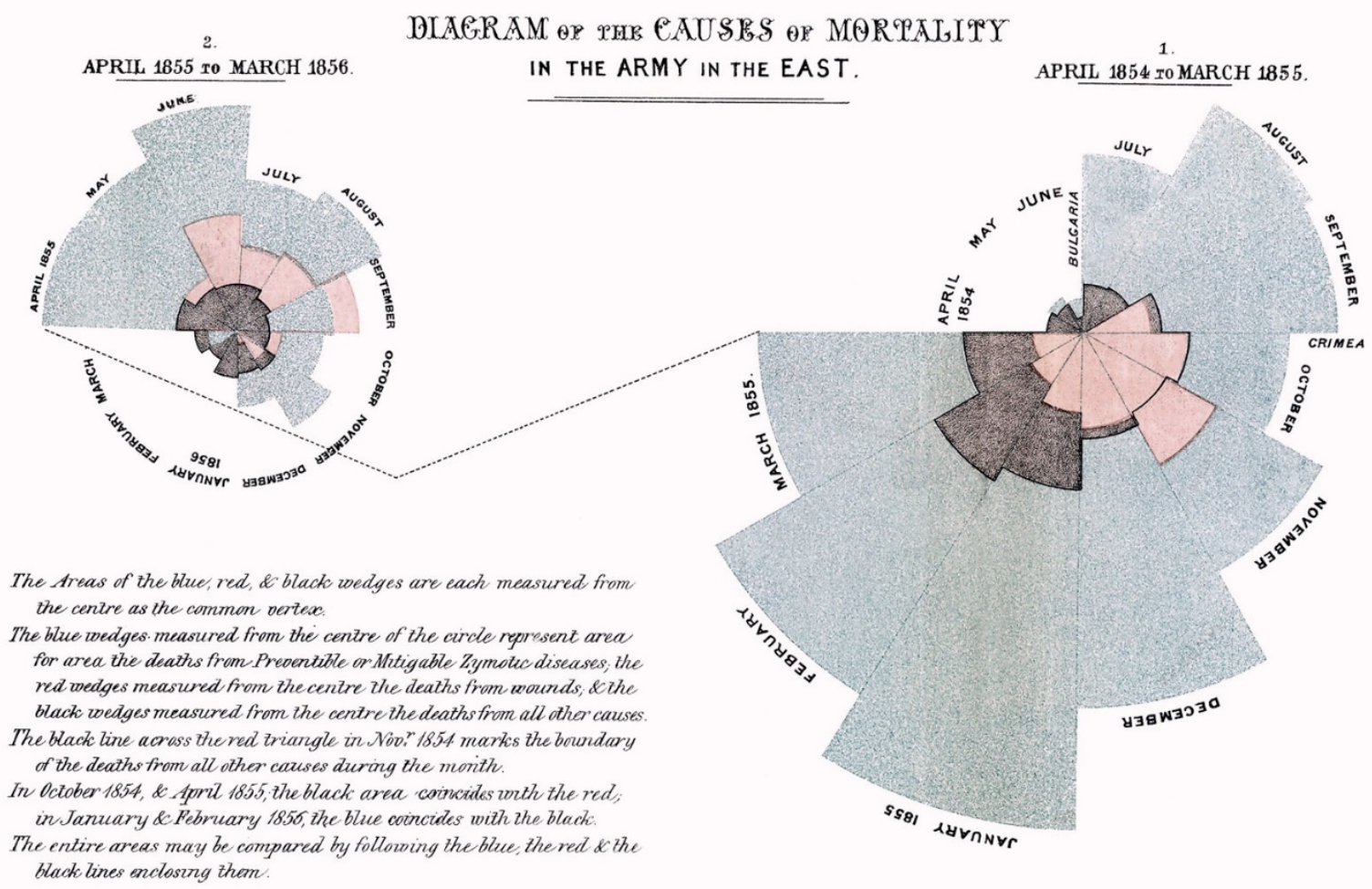

Figure 3. Nightingale rose diagram

Source: https://historyofinformation.com/images/3815a\%20Large.jpg

As teachers, we are aware that graphics would better engage students. The above examples illustrate that visual representations should play a prominent role in science teaching and learning not only because of the enhanced engagement, but also because visual representations are inseparable from scientific practice, knowledge, and communication.

\section{How do drawings and charts represent?}

There are many types of visual representations. This article focuses on two major types that are common in science education contexts. (i) Drawings. They include those that are drawn on paper or on computer screens, e.g., drawings of birds or trees in guides that facilitate identification, diagrams that represent submicroscopic particles or the water cycle. (ii) Graphs and charts. They include, typically, representations of relationships between variables in school science, such as the growth of population over time. In the media, graphs and charts take many different forms. Being able to make sense of them in the media is especially important for students to understand scientific issues that have strong social impacts (e.g., 'flattening the curve'). 
To tap into the potential of visual representations in research and teaching, it is useful to consider the way that they represent, namely through visuospatial mapping of their components with their referents (e.g., Tversky, 2011). The mapping may be based on likeness, in which the structural and spatial relationship of the components in a drawing resembles their referents (e.g., in the drawings of bird guides, the relative size of the beak and the bird head is roughly in proportion to real birds). On other occasions, the mapping is supported by the use of conventions. They include the use of colours, symbols, notations. Conventions are prominent in graphical representations, e.g., $x$ - and $y$ - axes, and are often inseparable from science practice (e.g., error bars, modelling the entire human circulatory systems as two connected circles). The mapping can be quite indirect in some conventions. For example, in the Nightingale rose diagram (Figure 3), the data for March and April 1855 are in separate charts. It is the dotted line that represents the temporal connection.

\section{Affordances of visual representations for communication}

The very nature of visual representations, that is, through likeness and conventions, provides affordances for communication that verbal representations such as written texts do not commonly have. Visual representations are powerful in a variety of contexts because they have some or all of the following three affordances (after Larkin \& Simon, 1987; Hegarty, 2011).

1) Multiple points of departure. Many visual representations do not have a fixed reading order that readers must follow. The reading order is more flexible than that of written texts. For example, readers can start by focusing on any features of a bird drawing in a bird guide based on their immediate need. The likeness between the drawing and the birds makes problem-solving with visual representations very efficient. In the Nightingale rose diagram, the casualties were represented by conventions (i.e., colours represented casualty categories and the size of the wedges represented the number of death), readers could start from any point in the diagram and they would still find that the blue wedges are bigger than the red wedges.

2) Efficient use of space. Besides the emotional appeal, the saying that 'a picture is worth more than a thousand words' is related to the feature that a visual representation can accommodate a huge volume of information within a limited space. For example, in the Nightingale case the key was to highlight the relative casualties of infections and of injuries (but less on the exact number of casualties). The monthly casualties were represented by the size of wedges (by conventions) but not by a table of numbers or verbal description. Similarly, it would have taken many words, if at all possible, to describe a moa in a way that they have the same details and precision as a drawing (by likeness). As another example, a London Tube map does not only represent stations, but also the way these stations are connected in different lines. The diagram is so efficient that it helps commuters, wherever they start and wherever they want to go, to navigate the complex system.

3) Making spatial relationships explicit and/or exact. This affordance relates more to drawings than charts or graphs. The likeness of drawings to their referents makes 
drawings more exact than words in representing spatial relationships. For example, the relative size of a bird and its beak is explicit and more exact in a drawing than in written texts. This is particularly important when a drawing refers to some unusual features (e.g., the size and shapes of Dodo and Kiwi beaks). As in a London Tube map, it makes very explicit the relative direction of stations, such that commuters could quite easily identify the platform (e.g., be it eastbound or westbound) that they should go to.

\section{Visual representations for the teaching and learning of science}

The affordances of visual representations create plenty of opportunities for their uses in teaching and research. This section discusses the use of student-generated drawings and graphics in the media to cultivate scientific literacy.

\subsection{Student drawings}

There is a body of literature in science education on utilising drawing to engage and support learning, and to probe into student understandings (Cheng \& Gilbert, 2015). In many science areas and concepts (e.g., the formation of geological features such as waterfalls and mountains, the structural-property relationship in chemistry, and the relationships between DNA, genes and chromosomes), precision in spatial relationships and visual features are key to understanding. The affordance of drawings, which make spatial relationships explicit and/or exact, render student drawings a valuable data collection tool when we want to investigate student understandings. This strategy provides an exactness of student understandings about spatial relationships and visual features that oral interviews do not easily do.

In classroom teaching, student drawings can support assessment for learning. These drawings provide important information about student understandings, which teachers can provide feedback upon. For example, in the teaching of the features of the gas state, it would be useful to ask students to observe and draw the steam from a boiling kettle. Although the mouth of the kettle is clear, it is quite common that students would draw otherwise (Figure 4). These drawings would create great opportunities for teachers and students to explore features of water at gas state (e.g., its opacity/colour with reference to the temperature of the clear zone and the steam). 

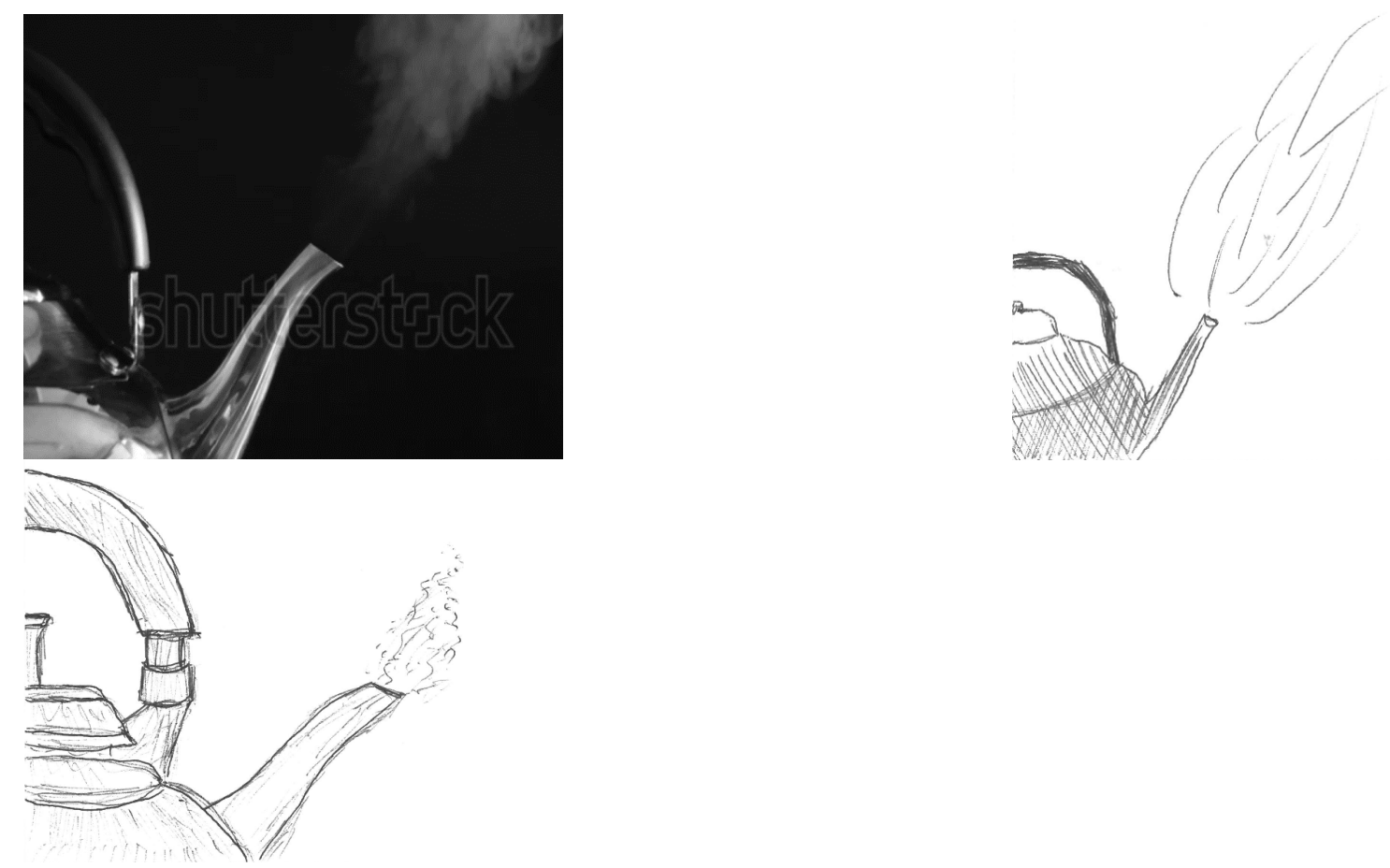

Figure 4. A boiling kettle and two drawings

Source: https://www.shutterstock.com/video/clip-23959360-tea-kettle-boiling-wateron-black-background

Classroom talks among students is an effective teaching strategy to support learning. While the exactness of spatial relationship and visual features allow students to draw to represent their ideas precisely, the multiple points of departure affords their peers flexibility to focus on different parts of the drawings and hence it is likely to open up opportunities for exploratory talks and inter-thinking. A recent study suggests that peerfeedback on student-generated drawing enhanced student understanding of chemical reactions (Galvez \& Cheng, 2020). We are now at the stage to produce scaffolds for students so that peer feedback can be even more fruitful for learning.

\subsection{Graphics in the media}

A key goal of science education is to cultivate scientifically literate citizens. Besides the schooling contexts, students are likely to learn science from the media. Beyond science, we also expect students to be able to engage in the media as a critical literacy practice (e.g., Leung \& Cheng, 2021). Media do not only use graphics and charts that we come across in school science, mathematics and statistics, but also create novel graphics to represent complex socioscientific phenomena. There is a need to harness these resources as teaching materials. A better understanding of what is entailed in making sense of these graphics would better support teachers in their planning.

The efficient use of space in visual representations means that there is a large volume of information presented in graphics in the media. While this affordance helps science communicators, this poses challenges to readers. Figure 5 reported the projections that three groups of scientists made respectively over different periods of 
time about the number of deaths from Covid-19. The lower bar charts show that the projection became more accurate on $4^{\text {th }}$ May 2020 as compared with that made on $12^{\text {th }}$ April 2020. There are plenty of conventions that readers need to unpack in order to make sense of these graphics (e.g., the meaning of the orange dots, the meaning of each line). Research studies have indicated that interpreting graphics demands relevant scientific content knowledge as much as graphical skills and practice. In this example, nevertheless, content knowledge and skills may not be enough. It is likely that readers would also need the following epistemic knowledge of science as they try to make sense of the graphics:

- Scientists made projections about future events

- The future is unknown. These projections are based on existing data. These projections are not always accurate. In the cases here, as they turned out, the projections were quite inaccurate at the early stage of the pandemic

- Different scientists could use different methods to make projections

- Scientists would revise their projections as time goes by

- Good projections mean their predictions are accurate

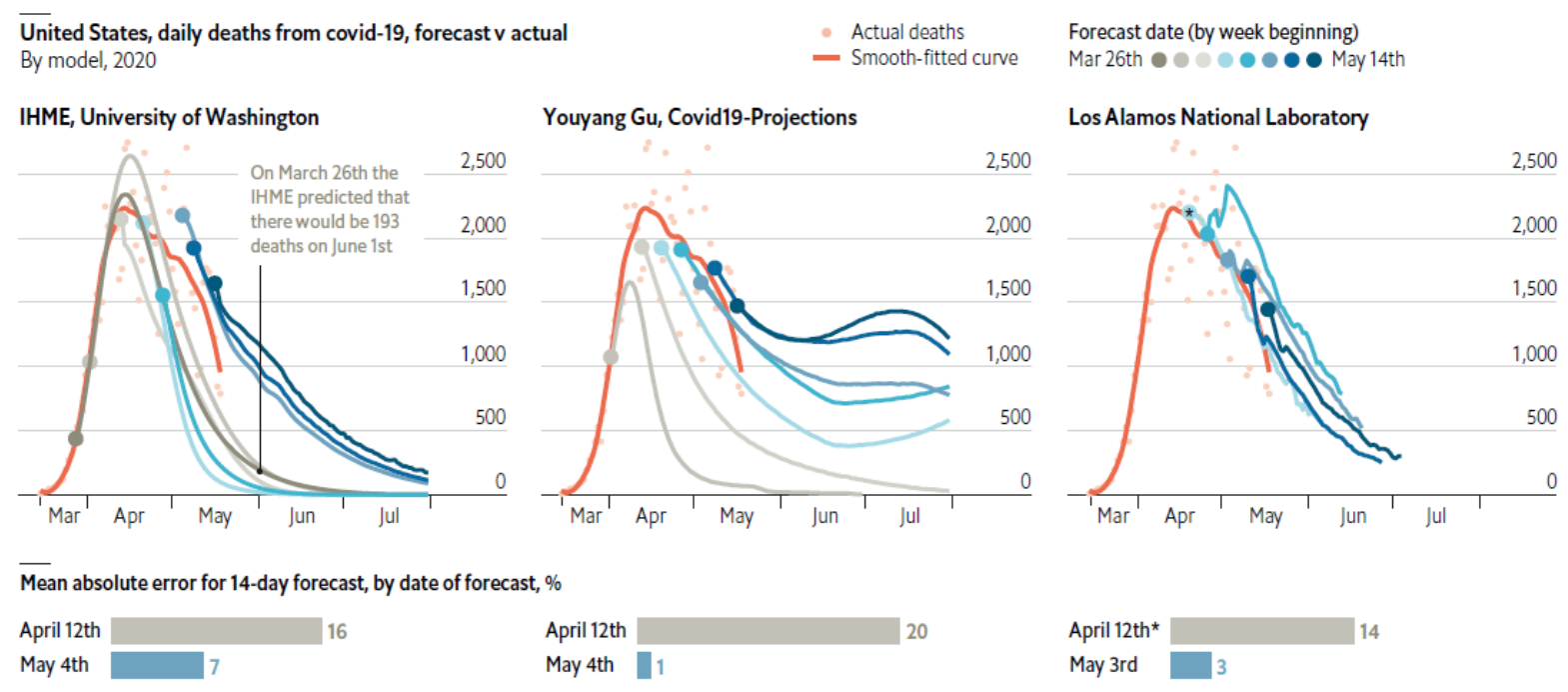

Figure 5. Projections of deaths from Covid-19

Source: https://www.economist.com/graphic-detail/2020/05/23/early-projections-ofcovid-19-in-america-underestimated-its-severity

The above analysis has the implication that these kinds of visual representations in media reports would support the teaching of the Nature of Science Strand of the New Zealand Science Curriculum in authentic contexts. In particular, it would help students to attain the 'Communicating in science' (how journalists and scientists make use of affordances of visual representations in communicating science) and 'Understanding about science' (how scientists work) Achievement Objectives. I would argue that developing students' capabilities and confidence in making sense of graphics and their conventions - those existing and those to be invented - is key in developing scientific literacy. And in this case, 
students would need a degree of understanding of epistemic practice of scientists (listed in the bullet points) in order to make sense of the graphics.

\section{Conclusion}

This paper posits that visual representations are key to scientific thinking and practice, and to science teaching and learning. There are three key affordances of visual representations. They make very efficient use of space in representing ideas, spatial relationships between objects in visual representations are explicit and exact; readers can have multiple points of departure when reading visual representations. These affordances provide us with a frame to consider the way that visual representations are useful for teaching, learning, and research. In classroom contexts, the spatial exactness and multiple points of departure allow readers (teachers or students) to provide specific feedback for conceptual development. Beyond formal science education contexts, there has been a surge of visual representations in the media. They take a variety of forms to represent a large volume of data within the space of a graphic. I attempt to make a case that this kind of visual representation can support the teaching of the Nature of Science, which is one of the keys to nurturing informed citizenship.

\section{Acknowledgements}

I would like to thank Denis Burchill and two anonymous reviewers who gave very useful feedback to the draft and diagrams of this paper.

\section{References}

Cheng, M. M. W., \& Gilbert, J. K. (2015). Students' visualization of diagrams representing the human circulatory system: The use of spatial isomorphism and representational conventions. International Journal of Science Education, 37(1), 136-161.

Galvez, G., \& Cheng, M. M. W. (2020). Cultivating Filipino students' submicroscopic understanding of a single replacement reaction via sketching and feedback synergism. Paper presented at the Australasian Science Education Research Association (ASERA), Wollongong, Australia, 24th - 26th June.

Hegarty, M. (2011). The cognitive science of visual-spatial displays: Implications for design. Topics in Cognitive Science, 3(3), 446-474.

Larkin, J. H., \& Simon, H. A. (1987). Why a diagram is (sometimes) worth ten thousand words. Cognitive Science, 11(1), 65-100.

Leung, J. S. C., \& Cheng, M. M. W. (2021). Trust in the time of corona: Epistemic practice beyond hard evidence. Cultural Studies of Science Education.

https://doi.org/10.1007/s11422-021-10045-9

Tversky, B. (2011). Visualizing thought. Topics in Cognitive Science, 3(3), 499-535. 
Maurice M.W. Cheng (PhD, KCL) is an Associate Professor of the Division of Education, The University of Waikato. His research interest is learning and teaching of science, in particular, on socioscientific issues and visual learning of scientific ideas. He has involved in Trends in International Mathematics and Science Study (TIMSS) for the past 15 years. He was the National Research Coordinator (Science) of TIMSS Hong Kong study 2019. He also serves in the Science Extended Expert Group for OECD's PISA 2024.

Email: maurice.cheng@waikato.ac.nz

ORCiD: https://orcid.org/0000-0002-8483-4074 